\title{
Muséologies
}

Les cahiers d'études supérieures

\section{Délibérer ou innover pour se réinventer : politiques des sciences et transformation des discours et projets institutionnels des centres de science et musées de la nature au Canada et en Ontario}

\section{Jonathan Paquette}

Volume 2, numéro 1, octobre 2007

URI : https://id.erudit.org/iderudit/1033595ar

DOI : https://doi.org/10.7202/1033595ar

Aller au sommaire du numéro

Éditeur(s)

Association Québécoise de Promotion des Recherches Étudiantes en Muséologie (AQPREM)

ISSN

1718-5181 (imprimé)

1929-7815 (numérique)

Découvrir la revue

Citer cet article

Paquette, J. (2007). Délibérer ou innover pour se réinventer : politiques des sciences et transformation des discours et projets institutionnels des centres de science et musées de la nature au Canada et en Ontario. Muséologies, 2(1), 14-28. https://doi.org/10.7202/1033595ar
Résumé de l'article

Une décennie, c'est le temps qu'il aura fallu pour observer une évolution de l'attitude du public à l'égard des sciences et voir se manifester un intérêt de la part des gouvernements pour les musées et les centres de science. L'accès à l'information et l'éducation sont alors devenus des enjeux important pour ces institutions, favorisant ainsi la mise en place de nouveaux plans de communication. L'article qui suit fait une mise au point de la situation actuelle des choix institutionnels quant aux politiques, et aux discours de ces musées. 


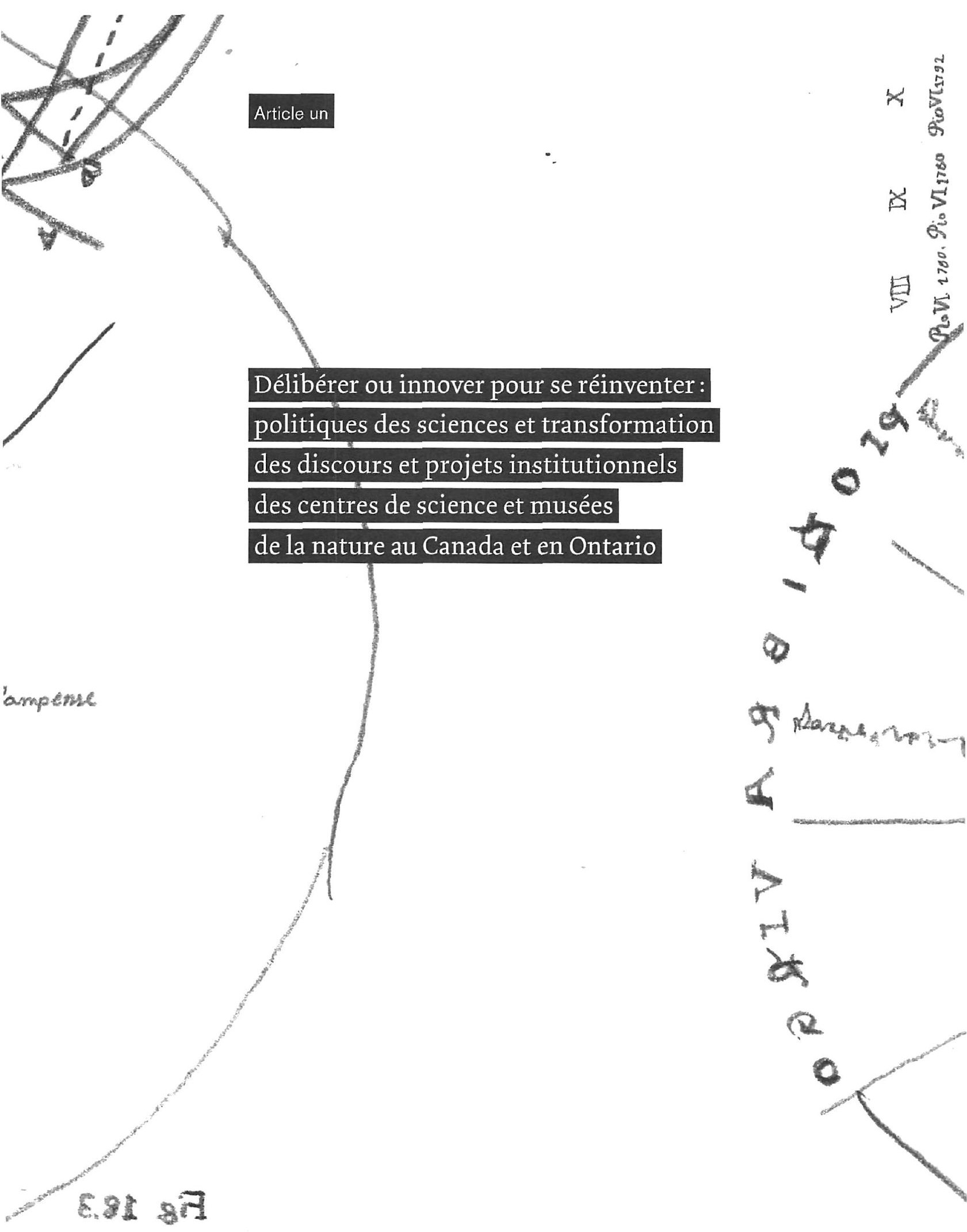


Une décennie, c'est le temps qu'il aura fallu pour observer une évolution de l'attitude du public à l'égard des sciences et voir se manifester un intérêt de la part des gouvernements pour les musées et les centres de science. L'accès à l'information et l'éducation sont alors devenus des enjeux important pour ces institutions, favorisant ainsi la mise en place de nouveaux plans de communication. L'article qui suit fait une mise au point de la situation actuelle des choix institutionnels quant aux politiques, et aux discours de ces musées.

JONATHAN PAQUETTE EST CANDIDAT AU DOCTORAT À LÉCOLE NATIONALE D'ADMINISTRATION PUBLIQUE. DEPUIS 2005, IL EST MEMBRE DU CORPS PROFESSORAL DE SCIENCE POLITIQUE ET D'ADMINISTRATION PUBLIQUE ET PROFESSEUR À LA FACULTÉ DES SCIENCES SOCIALES DE L'UNIVERSITÉ LAURENTIENNE, SUDBURY (ONTARIO). CHERCHEUR RÉGULIER AU CENTRE FOR LOCAL GOVERNMENT, SES RECHERCHES PORTENT NOTAMMENT SUR LES POLITIOUES CULTURELLES. 
DI Cocco, Caroline,

ministre de la Culture de

I'Ontario. Communiqué de presse. Ministère de la Culture de l'Ontario, 26 juillet 2006.

[2]

CASTELLS, Manuel. L'ère de l'information. Paris : Fayard, 1998

[3]

UNESCO.

Vers les sociétés du savoir. Paris : Éditions de l'UNESCO, 2005, p. 27-29.
En raison du passage d'une politique des sciences à une politique formelle de l'innovation en 2002, le domaine de la "culture scientifique " au Canada a perdu ce qui pouvait lui rester de légitimité. En effet, un important programme de financement, Science et Culture Canada, était annulé en 1999, assénant aux professionnels du secteur un coup difficile à encaisser. À la fin des années 1990, la "culture scientifique " n'est plus à l'ordre du jour. Malgré tout, ce qui se présente comme un ensemble de contraintes se révélera comme une opportunité pour les praticiens qui œuvrent dans le secteur de la muséologie scientifique et technique. Alors que plusieurs pays de l'OCDE (Organisation de coopération et de développement économiques) se lancent dans la formulation de politiques de l'innovation, laissant de côté les politiques des sciences traditionnelles, le climat des rapports entre science et société prend un tournant différent. Au début des années 2000, on assiste également à une médiatisation accrue des craintes à l'égard des technosciences. De la plus optimiste Déclaration de Vancouver en 1989 au nouvel Agenda pour la science de l'Unesco en 1999, il aura suffi de dix ans pour assister à un changement d'attitude du public à l'égard des sciences. Dans un tel contexte, nous pouvons observer l'émergence d'un intérêt affirmé par les différents gouvernements envers les musées de science.

Stimulé par un rapport science / société plus ambigu, plusieurs pays se tournent vers les musées de science, les musées de la nature et les centres de science afin de mettre en œuvre des plateformes de communication diverses. En Europe, l'avènement du nouveau millénaire est accompagné d'une volonté de renouveler la gouvernance des sciences qui se présentera avec beaucoup plus d'acuité en raison de la diffusion des thèses de la société de l'information ${ }^{[2]}$. Les enjeux de l'accès à l'information, de l'éducation et de la participation se sont taillés une place imposante au sein des programmes électoraux ${ }^{[3]}$. Envahissantes, les thèses de la société de l'information et du savoir se sont constituées en un référentiel partagé par différentes instances gouvernementales et de multiples acteurs sociaux. En conséquence, 
c'est la conception d'un lien social fondamentalement communicationnel qui oriente les stratégies et les choix publics, initiant du coup l'abondance d'une forte demande d'espaces pour communiquer. Sur l'initiative des instances de l'Union européenne et de plusieurs États tels la France et la GrandeBretagne, les musées de science et plus particulièrement les centres de science sont courtisés afin d'offrir des solutions en matière de communication des sciences. Plus simplement, certains musées offrent leur espace à des expériences de démocratie délibérative pour discuter de thèmes aussi actuels, techniques et controversés que ceux des OGM (organismes génétiquement modifiés), des xenotransplantations ou encore des nanotechnologies. Sous l'impulsion de l'Union européenne en 2004 s'amorcent les Meeting of Minds, série de réunions et de séances de délibération sur les neurosciences qui ont pour partenaires des musées aussi prestigieux que la Cité des sciences et l'industrie de Paris. Cette pratique partenariale est exemplaire de cette nouvelle alliance qui se tisse entre les musées de science et les logiques communicationnelles.

Ces changements ne sont pas les seuls et les institutions de la muséologie scientifique et technique constituent un territoire vers lequel convergent également bon nombre de mutations dans les rapports entre l'État et la culture. Aussi retrouve-t-on les musées associés à une pluralité de projets récréotouristiques et de développement local. Plus particulièrement, les musées sont assujettis depuis les années 1980 à un certain nombre de restrictions budgétaires qui ne font que participer au renforcement des stratégies de management public, privilégiant les attitudes commerciales, le retour sur investissement et la prédominance d'une conception du musée comme loisir au détriment des missions publiques, notamment de la recherche. Selon Anne Gombault ${ }^{[4]}$, toutes ces transformations aident à créer une crise d'identité organisationnelle au sein des musées. Ces changements multidirectionnels, au confluent de la nouvelle gestion publique et d'un contexte nouveau de la communication des sciences, contribuent certainement à la crise et aux tentatives de renouveau identitaire des musées de science et .technologie au pays. Malgré toutes ces tensions institutionnelles, les musées de science, les centres de science et les musées de la nature attirent toujours un important public. À titre d'exemple, au Québec, les musées de science et les centres d'interprétation des sciences accueillaient respectivement pas moins de 2921672 et de 1604023 visiteurs en $2006^{\text {[s]. }}$

Dans certains musées et centres de science de tutelle publique en Ontario et de niveau fédéral au Canada, on assiste à l'émergence d'un discours de changement. Au tournant des années 1990-2000, quatre des plus grands musées de science et technologie du pays ont pris le sentier de la "réinvention". Ces discours peuvent même être nommés par des énoncés associés au changement institutionnel : Renaissance ROM (Royal Ontario
[4]

GOMBAULT, Anne.

"La nouvelle identité organisationnelle des musées: Le cas du Louvre». Revue française de gestion, vol. 29, no 142, 2003, p. $170-171$.

[5]

OBSERVATOIRE DE LA CULTURE ET DES COMMUNICATIONS DU QUÉBEC.

Statistiques principales de la culture et des communications au Québec. Québec: Gouvernement du Québec, 2007, p. 46. 
[6]

FAIRCLOUGH, Norman. Critical Discourse Analysis. The Critical Study of Language. Londres : Longman, 1995.

[7]

ZAN, Luca "Managerialisation Process and Performance in Arts Organizations: the Archeological Museum of Bologna". Scandinavian Journal of Management, vol. 16, 2000, p. 433.

[8]

Ibid.

[9]

LÉGARÉ, Benoît "La muséologie scientifique un terreau expérimental. Portrait des tendances et prospectives" In. BERGERON, Yves (dir.). Musées et muséologie: Nouvelles frontières.

Essais sur les tendances.

Québec: Musée de la civilisation - Société muséale du Québec, 2005
Museum / Musée royal de l'Ontario); Opération innovation(Centre de science de l'Ontario); Rénovation / Renouvellement (Musée de la Nature); le Grand Changement (Science Nord). Qu'en est-il des discours de "renouvellement ", de « réinvention» ?

À partir d'une analyse de discours institutionnels ${ }^{[6]}$ fondée sur l'étude des productions et des communications organisationnelles, nous proposons d'apporter un éclairage sur la question afin de saisir les directions actuelles et les choix institutionnels des musées de science et technologie à l'étude. De toute évidence, les cas abordés se rattachent à des pratiques muséologiques diverses allant du musée de la nature au centre de science, en passant par des projets interdisciplinaires tel celui du Musée royal de l'Ontario. Ces nuances dans les pratiques muséologiques annoncent des trajectoires institutionnelles différentes, voire divergentes.

\section{La réinvention : un "genre " issu du management public}

Le vaste chantier de réformes administratives du secteur public que l'on situe au tournant des années 1970 et 1980 se décline en une pluralité de projets tantôt complémentaires, tantôt contradictoires. Si certaines stratégies de réforme s'intéressent principalement aux structures et aux modes de livraison des services publics (privatisation, contractualisation, partenariats public-privé / PPP), certaines stratégies, tout aussi idéologiquement orientées, chercheront à mobiliser les valeurs et les projets institutionnels de différents organismes publics. De la vague des transformations administratives du secteur public, le domaine de la culture n'est pas en reste. Le managérialisme est une pratique qui touche les organisations du secteur de la culture, notamment les musées, depuis bon nombre d'années ${ }^{[7]}$. Ce phénomène managérialiste apparaît dans les musées comme un discours qui fait rupture avec les logiques traditionnelles (conservation) de l'institution. Le conflit entre les logiques professionnelles établies et la logique managériale est alimenté par le primat " organisationnel » et économiste de cette logique managériale ${ }^{[8]}$. En fait, dans le secteur culturel, le management apparaît comme un discours qui valorise les logiques marchandes et mesure le succès en termes de profit, sinon de "visites » et de fréquentation du musée, au détriment d'une logique institutionnelle tournée vers le service public, la préservation, la recherche et la communication du patrimoine. Devant ces nouvelles exigences, les musées de science s'engagent dans certains sentiers et explorent leurs frontières muséologiques ${ }^{[9]}$. 
Les réformes administratives sont généralement accompagnées, annoncées ou stimulées par une panoplie de discours appelant, voire célébrant le changement. Selon Christopher Pollitt et Geert Bouckaert ${ }^{[10]}$, le langage des réformes de nouvelle gestion publique est chargé de sens et de nuances en plus d'être fortement idéologiquement connoté. Or, le " genre ${ }^{[11]}$ " discursif de la réinvention naît et se popularise dans les années 1990 sous la plume de David Osborne et Ted Gaebler ${ }^{[12]}$. Célèbre ouvrage associé au projet de réforme des services publics aux États-Unis, Reinventing Government présente, à la manière des ouvrages normatifs de l'administration publique des années $1930^{[13]}$, un ensemble de principes qui pourraient contribuer à établir un gouvernement et une administration plus efficaces. Cette efficacité en question est entendue comme étant possible par le truchement d'une administration non bureaucratique, mais plutôt entrepreneuriale. L'ouvrage traduit bien le style des écrits normatifs liés à l'appel aux réformes de nouvelle gestion publique. Parmi les principes prônés par Osborne et Gaebler, on trouve notamment les notions d'habilitation et de prise en charge de la collectivité, de compétition, de gestion par résultats, de service au client, de gestion participative, l'esprit d'innovation et de profitabilité du service public. Manifeste de nouvelle gestion publique, l'ouvrage somme par ailleurs les organisations publiques de se prendre en main.

De ce que nous pouvons comprendre a priori, les discours de nouvelle gestion publique sont des appels à l'action, à la transformation de la nature du service public. Au-delà des changements plus structurels, on peut identifier un certain nombre d'énoncés qui visent directement les valeurs et les acteurs du secteur public. La "réinvention " convoque au changement et son discours cherche à instaurer un nouveau régime de la vérité au sein des organisations publiques. Autrement dit, aux côtés des discours de "réforme", de réingénierie et de modernisation, les discours de réinvention constituent un genre discursif qui, dans sa spécificité, valorise notamment les valeurs de l'entrepreneuriat que sont celles du risque et de l'initiative. Au-delà de la structure discursive typique à cette stratégie et à ce style de changement dans le secteur public, il faut se tourner vers le contexte plus spécifique aux musées de science, qu'est celui des transformations récentes de la gouvernance des sciences. Dans le sillage de la formulation d'une politique de l'innovation se trouve le ferment d'une remise en question de la légitimité de la culture scientifique, comme culture savante et citoyenne de l'amateur des sciences ${ }^{[14]}$.

\section{[10]}

POLLITT, Christopher et Geert Bouckaert. Public Management Reform. A Comparative Analysis. Oxford : Oxford University Press, 2004, p. 18. [11]

CZARNIAWSKA, Barbara. Writing Management. Organization Theory as a Literary Genre. Oxford : Oxford University Press, 1999, p. 11-12. [12]

OSBORNE, David et Ted Gaebler. Reinventing Government. How the Entrepreneurial Spirit is Transforming the Public Sector. New York : AddisonWesley, 1992.

[13]

Nous faisons référence ici aux productions savantes produites pendant la période 1926-1937, qui constituent des piliers de l'administration publique classique. Voir HENRY, Nicholas "Paradigms of Public Administration ", Public Administration Review, vol. 35, no 4, 1975, p 378-386.

[14]

Sur le sujet, voir notamment SCHIELE, Bernard. Quand la science se fait culture. La culture scientifique dans le monde. Québec : Éditions Multimonde, 1994.

On peut également consulter Donghong, Cheng, Jenni Metcalfe et Bernard Schiele. At Human Scale. International Practice in Science Communication. Beijing : Science Press, 2006. 
Des politiques de science aux politiques de l'innovation

Suivant l'élection qui a mené au pouvoir les libéraux de Jean Chrétien en 1993, le nouveau gouvernement entreprend une révision des politiques et des

[15]

CHAMBERLIN, Tyler et John De la Mothe. "The Integration of Innovation Policies : The Case of Canada". Prometheus, vol. 22, no 1 , 2004, p. 4.

[16]

Ibid.

[ 7$]$

GODIN, Benoit.

La science sous observation. Cent ans de mesure sur les scientifiques : 1906-2006. Québec : Les Presses de I'Université Laval, 2006.

[18]

INDUSTRIE CANADA. Avis en sciences et technologies - Au-delà du cadre établi. Rapport sur les activités fédérales en science et en technologie. Ottawa : Gouvernement

du Canada, 2002, p. 6.

[19]

LEMELIN, André

Le soutien public à la culture scientifique et technique dans quelques États. Rapport d'expertise conseil produit pour le Gouvernement du Québec. Québec : Les productions de l'espace-temps inc., 2002.

[20]

ELAM, Mark et Margareta Bertilsson. "Consuming, Engaging and Confronting Science. The Emerging Dimensions of

Scientific Citizenship".

European Journal of Social

Theory, vol. 6, no 2, 2003.

[21]

SELECT COMMITTEE ON SCIENCE AND TECHNOLOGY. Science \& Society. Londres : House of Lords, 2000. programmes fédéraux en matière de science et technologie ${ }^{[15]}$. La révision qui se déroulera en 1994-1996 donnera lieu à la politique fédérale de l'innovation qui sera officiellement rendue publique en $2002^{[16]}$. Comme plusieurs pays de l'OCDE préoccupés par les impacts économiques des sciences et technologies, c'est dans la voie de l'innovation que le Canada s'inscrira ${ }^{[17]}$. Cette transition d'une politique de la science et de la recherche en une politique de l'innovation consacre l'importance de la dimension économique et commerciale qui prendra désormais le dessus dans les activités de recherche. En annonçant une politique de l'innovation en 2002, le gouvernement fédéral mise sur le développement d'une économie compétitive, le développement des compétences et l'accélération de la commercialisation des innovations technologiques ${ }^{[18]}$. Cette transformation de la politique scientifique témoigne d'une attitude différente du gouvernement fédéral en matière de science. Autrement dit, l'idée de la science pour la science ne suffit plus, la nouvelle politique fédérale de l'innovation mise sur la science et la recherche pour le développement économique et commercial.

Ce changement de politique annonce un cadre culturel et cognitif nouveau qui met au défi les conceptions plus romantiques et désintéressées de la communication des sciences. En 1999, Science et Culture Canada, le seul programme fédéral de communication des sciences, est aboli, malgré son évaluation positive lors de l'exercice d'examen de programmes ${ }^{[19]}$. Au-delà du désaveu que cette décision constitue pour les acteurs de la culture scientifique, cela représente un important changement de mentalité dans les rapports entre le gouvernement fédéral et le domaine de la communication des sciences. Davantage tournés vers le modèle communicationnel, les musées de sciences et les centres de science se montrent plus perméables, voire des acteurs attentifs aux nouveaux besoins du gouvernement du Canada et, plus récemment, du gouvernement de l'Ontario. Le leadership en la matière de l'Association canadienne des centres de science atteste d'une certaine énergie consacrée à l'établissement d'un partenariat entre les musées et la construction d'une culture de l'innovation au pays.

Alors que le Canada et certains pays d'Europe, à l'instar de l'Australie et de la Nouvelle-Zélande, se lancent dans la formulation de politiques de l'innovation, des rapports science / société peu harmonieux précipitent les échanges et les commandes d'intervention des partenaires muséaux. Lémergence de nouvelles technologies du vivant et les conséquences des innovations scientifiques et techniques sur l'environnement contribuent à créer un climat de méfiance à l'égard des sciences ${ }^{[201}$. Moment emblématique de ce changement d'opinion publique, en février 2000 un rapport spécial du 
Comité des sciences et technologies de la Chambre des Lords de la GrandeBretagne indique que les rapports entre la science et la société vivent une "phase critique» et que la "science a perdu son droit à la pratique " ${ }^{[21]}$. Au Canada, on voit des prises de conscience similaires. Les rencontres menant à la participation du Canada à la Conférence mondiale sur la science de l'UNESCO ${ }^{\text {(22) }}$ de 1999 attestent d'un changement de "climat " à l'endroit des sciences; on souligne parmi les citoyens une attitude plus ambivalente : "Au sein du public, on décèle une certaine méfiance, voire crainte, de la science et de la technologie. Cela témoigne d'une importante lacune de communication entre les scientifiques et la société ${ }^{[23]}$ ", alors que dix ans auparavant, la réflexion faisait figure de prise de conscience des limites de la science. Un besoin de renouvellement des médiations science et société s'installe et les musées de science, de la nature, mais surtout les centres de science sont sollicités afin de prendre part à ce renouvellement.

Sur le plan des pratiques muséologiques, là où la logique communicationnelle prédomine, le besoin de renouveler les rapports entre science et société coïncide également avec certaines limites associées à la pratique de la communication des sciences. Si l'utilisation du musée de sciences comme instrument de communication rend compte d'un besoin particulièrement saillant pour les gouvernements, il demeure que la pratique de la communication des sciences est elle-même mise au défi. En effet, le paradigme traditionnel de la communication publique des sciences semble aussi avoir été mis à l'épreuve par la perte de confiance du public à l'égard des sciences. À la fin des années 1990, les mesures d'opinion publique, tel l'Eurobaromètre, identifient le glissement dans l'opinion publique en question. Egil Kallerud et Inge Ramberg ${ }^{[24]}$ en concluent qu'une population ayant un fort développement de connaissances scientifiques tend davantage à avoir une attitude "négative» à l'égard des sciences et des innovations. En somme, les résultats récents de l'Eurobaromètre portent à croire que le projet traditionnel de la communication des sciences, à savoir que la communication et la transmission de connaissances entraînent l'acceptation sociale des sciences, s'avère inopérant. Autrement dit, le paradigme dominant de la communication des sciences est secoué. Cela dit, les centres de science et les musées de science ne sont pas seuls à devoir repenser leur rapport avec le patrimoine scientifique. Pour les musées de la nature, l'apparition de nouvelles pratiques scientifiques dans le domaine des biosciences est en mesure de stimuler les projets institutionnels et les pratiques muséologiques de ces musées. La biologie et les sciences du vivant se détournent des spécimens pour s'orienter vers des objets beaucoup moins aptes au collectionnement. Les approches communicationnelles, le design et les représentations deviennent en ce sens des pratiques beaucoup plus attrayantes, mais aussi de nouveaux points de repères pour les professionnels. Bref, le passage aux politiques de l'innovation a créé un contexte d'opportunités pour les acteurs de la muséologie scientifique.
La déclaration de Vancouver. Actes de Colloque de I'UNESCO. La science et la culture au XXIe siècle, un programme de survie. Paris : UNESCO, 1989. [23] UNESCO. Agenda pour la science. Cadre d'action.

Paris : UNESCO, 1999. [24] KALLERUD, Egil et Inge Ramberg. "The Order of Discourse in Surveys of Public Understanding of Sciencen. Public Understanding of Science, vol. 11, 2002. 
[25]

MINISTÈRE DES COMMUNICATIONS. Rapport du Comité d'étude de la politique culturelle fédérale. Ottawa : Gouvernement du Canada, 1982, p. 96.

[26]

Idem, p. 97.

[27]

MUSÉE CANADIEN DE LA NATURE. Projet de renouvellement : vision du Musée canadien de la nature. Ottawa : Gouvernement du

Canada, 2006.

[28]

MUSÉE CANADIEN DE LA NATURE. Virage et renouvellement. Rapport annuel de 1997-1998, Ottawa : Gouvernement du Canada, 1998 ; et Musée canadien de la nature. Le renouvellement d'un trésor naturel. Sommaire d'un plan d'entreprise 2005-2006.

Ottawa : Gouvernement du Canada, 2006.

[29]

MUSÉE ROYAL DE L'ONTARIO. Annual Report 2001/2002. Toronto: Gouvernement de l'Ontario, 2002.

\section{Confession managériale}

Au Canada, les auteurs du rapport du comité Applebaum-Hébert sur la révision de la politique culturelle fédérale de 1982 constatent que les ressources n'ont pas augmenté et que l'engagement financier de l'État était plutôt faible : "les établissements canadiens sont actuellement incapables de conserver, de cataloguer ou d'exposer convenablement le matériel qu'ils détiennent " ${ }^{[25]}$. Le rapport Applebaum-Hébert est annonciateur d'une attitude différente à l'égard des musées sur le plan fédéral en ce qu'il suggère que ceux-ci en sont à un point tournant et qu'ils doivent « accepter les limites de la croissance " ${ }^{[26]}$. On peut observer un phénomène similaire du côté des institutions muséales de la province de l'Ontario, rapidement entrée dans le sentier d'une municipalisation des appareils culturels quelques années seulement après la création du ministère des Affaires culturelles et des loisirs en 1975. Lévolution des politiques muséales laisse croire à un détachement de la régulation de l'offre culturelle par l'État au profit d'instruments qui valorisent la conception organisationnelle des musées, contribuant notamment à la managérialisation des musées et à la justification, voire à la professionnalisation des pratiques managériales.

Tirant parti de l'inspiration générée par cette nouvelle gestion publique, les discours de réinvention des musées de science et de technologie problématisent l'institution en fonction de lacunes dans la gestion des opérations. Cette problématisation de l'institution apparaît alors comme l'énoncé initial qui habilite et légitime le chantier du renouveau. Le renouvellement du Musée canadien de la nature fait état du besoin pressant d'augmenter les recettes et d'arrimer les recherches à la diffusion grand public ${ }^{[27]}$. Autrement dit, le musée s'engage à corriger le tir et entrera dans l'économie des industries du loisir de la région de la capitale nationale, logique de compétition que la décentralisation des musées prévue par la politique fédérale de 1990 n'a apparemment pas été en mesure de réaliser pleinement ${ }^{[28]}$. On trouve également cette caractéristique du discours dans plusieurs autres musées de science et de technologie. Les communications managériales entourant le renouvellement du ROM reprennent les traits de la «cure " économique et financière en promettant de stimuler sa mission et de doubler ses revenus de recettes dans les prochaines années : «Renaissance ROM is the essential instrument in rebuilding the ROM's economic health ${ }^{[29]}$. " En somme, l'examen des communications institutionnelles et des pratiques discursives mobilisées par les acteurs de l'administration laisse croire à l'importance que ce type d'énoncés peut jouer dans le renouvellement des musées de science et de technologie.

Pour ces années de disette dans certains secteurs de la culture, l'énoncé de la « confession» facilite la problématisation du musée en tant qu'organisation 
à gérer plutôt qu'un patrimoine à préserver et à valoriser. Ces formations discursives annoncent certainement un apprentissage, par les directions, de différentes stratégies de médiation entre musée et gouvernement. Le développement des musées, la quête d'espaces et le financement des projets d'infrastructure doivent pouvoir annoncer un retour sur l'investissement. Or, ces discours au ton thérapeutique laissent à penser que les musées en question nécessitent une cure majeure pour accroître l'efficacité et l'efficience de leur gestion. Tous les éléments ici sont réunis pour que l'on puisse parler de véritables confessions dans lesquelles linstitution est assujettie au primat managérialiste et où «faute avouée est à demi pardonnée». Cette confession d'un passé peu envieux et la promesse d'un passé rentable sont elles-mêmes accompagnées d'une nouvelle problématisation de linstitution muséale dans ses rapports au patrimoine naturel, scientifique et technique.

Conversion scientifique

Les communications organisationnelles et les plans de développement des musées s'appuient sur une nouvelle vision du secteur. Depuis 2002, l'Association canadienne des centres de science est plutôt active dans l'effort de consolidation des partenariats entre le ministère de l'Industrie et les centres de science, afin qu'ils puissent être des acteurs essentiels de la nouvelle "culture de l'innovation ". Il en va de même avec l'Ontario, qui s'est récemment tournée vers cette idée. Ce changement de "culture" n'est pas seulement attribuable à des stratégies propres aux centres de science. En fait, la province de l'Ontario, à l'heure de sa propre initiative en matière d'innovation, encourageait fortement une telle transition; c'est ce qu'atteste un communiqué de presse du Centre des sciences de l'Ontario (CSO), citant l'étonnement de la ministre de la culture (Caroline Di Cocco) : «La province se réjouit du changement de philosophie du Centre des sciences et de son leadership dans la création d'activités livrées dans un lieu qui encourage une plus grande participation du public ${ }^{130}$. " Dans les centres de science de l'Ontario, la réinvention implique un passage d'une culture d'amateur des sciences à une culture caractérisée par le risque, linnovation, les compétences

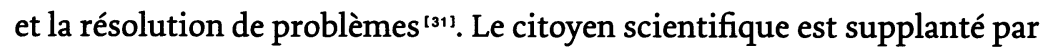
l'apparition d'un visage nouveau, celui de l'entrepreneur.

Pour une institution muséale plus traditionnelle, comme le Musée royal de l'Ontario (ROM), la transition d'une culture scientifique à une culture de l'innovation est plus nuancée, considérant la place distincte que la muséologie de la nature accorde à la communication. Cela dit, une des premières phases de la «Renaissance» du ROM a consisté en une fusion rassemblant
[30]

MINISTÈRE DE LA CULTURE. Le Centre des sciences de I'Ontario transformé par l'Opération Innovation. Communiqué de presse, 26 juillet 2006. Toronto : Gouvernement de l'Ontario.

[31]

CENTRE DES SCIENCES DE L'ONTARIO. The Agents of Change Initiative. Innovate. News, Spring/Summer 2006. Toronto : Gouvernement de l'Ontario, 2006. 
[32]

MUSÉE ROYAL DE L'ONTARIO.

Transition et croissance. Rapport annuel 2003-2004. Toronto : Gouvernement de I'Ontario, 2004.

[33]

MUSÉE ROYAL DE L'ONTARIO. Annual Report 2001/2002. Toronto : Gouvernement de I'Ontario, 2002.

[34]

LATOUR, Bruno. Nous n'avons jamais été modernes.

Paris : La Découverte, 1989 ; et Stengers, Isabelle. L'invention des sciences modernes. Paris : La Découverte, 1993. de nombreuses unités de conservation et de recherche ${ }^{[32}$ dans le but de consolider le projet institutionnel du ROM sur deux piliers, la nature et la culture, laissant de côté l'histoire, mais habilitant un dialogue entre nature et culture ${ }^{[33]}$. On retrouve dans le discours de la "Renaissance» le souci de réintégrer le public, d'offrir un espace de discussion des enjeux de société, mais aussi d'assumer un leadership en matière d'éducation. Plus visiblement dans le cas du ROM, on peut identifier un renouveau dans la possibilité de produire un discours sur la science et la société, une transformation, à tout le moins sur le plan organisationnel, de la configuration des producteurs de discours sur la science et, conséquemment, une modification des conditions de la pratique discursive.

On trouve également un passage, un changement d'une culture scientifique à une culture del'innovation, chez Science Nord. Alors que les centres de science s'orientent vers la pédagogie de l'innovation, les musées scientifiques à composante muséologique traditionnelle (nature) sont plutôt axés vers l'ouverture du dialogue science et société. Le "renouvellement » du musée de la nature se poursuit dans un recentrage institutionnel qui répond aux temps présents : « Dans toutes nos activités contemporaines, du jardinage à l'épissage de gènes, les interactions de la société humaine et de la nature sont à la fois constantes et inéluctables. La raison d'être du Musée canadien de la nature consiste à faire comprendre aux gens cette interdépendance ${ }^{[34]}$. " Plus récente au musée de la nature, la logique communicationnelle qui semble prendre assise au sein de ces institutions s'oriente sensiblement dans le sillon des théories de la démocratie délibérative, signe que la communication prend une place envahissante au sein de l'institution. Le sentier du " Parlement des choses ${ }^{\text {(35) }}$ " qui ferait du musée de la nature un espace de délibération sur l'avenir de la science et de la société permet à ce genre de musée de renouveler son rapport aux objets. Loin de la période faste des explorations et des voyages exotiques du XIX ${ }^{e}$ siècle, certains objets biologiques ou sociotechniques aujourd'hui sont plus difficilement collectionnables. Or, alors que les musées de science et les centres de science se font les partenaires de la culture de l'innovation, du côté des musées de la nature l'approche de réinvention s'appuie sur les outils de la communication et du design, ce qui permet, comme dans le cas du centre de science, de resocialiser la science dans son espace. La table est dressée pour un recentrage de l'institution, passant de la recherche traditionnelle des spécimens aux recherches des rapports science / société inspirées des sciences humaines. 


\section{Se réinventer et traduire : entre pouvoir et identité}

L'analyse de discours en administration publique dépasse largement les aspects idéationnels pour inclure les pratiques discursives ainsi que les modes de consommation, de reproduction et de circulation des discours. Derrière les changements organisationnels, les changements de structures, d'infrastructures et de pratiques, on trouve une panoplie de discours et de savoirs experts, parfois très locaux, cherchant à instaurer un « régime de la

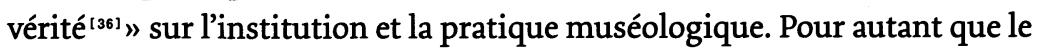
genre de la réinvention puisse représenter une possibilité de transformations et de contrôle des organisations publiques, le pouvoir n'est jamais total, ni pure domination, et les discours peuvent à leur tour devenir des espaces de résistance et de négociation. Chacun des projets de réinvention institutionnelle examiné s'appuie sur des projets d'infrastructures et sur des potentielles réformes de l'organisation et du fonctionnement des musées respectifs. Il est intéressant de noter à ce sujet que les discours de réinvention se situent à la jonction entre les besoins criants des institutions de la muséologie scientifique et le désir de rentabiliser ces musées. Les « confessions " que nous retrouvons dans les différents discours permettent de faire le pont entre les réticences des gouvernements à investir et la promesse d'une rentabilité future et possible par des projets de transformation des infrastructures des musées.

Que faire quand la culture n'est pas au programme et que faire pour intéresser les différents paliers de gouvernement aux musées quand personne ne s'y intéresse vraiment ? Dans le cas de l'Ontario, on pourrait ajouter une autre question : que doivent faire les acteurs des musées pour mettre fin à un gel du financement des opérations qui dure depuis plus de dix ans? L'analyse de discours institutionnels permet de révéler des usages des formations discursives de réforme qui sont plus émergentes et qui prennent assise au sein même des organisations publiques. Pour intéresser les décideurs aux musées, il faut faire quelques détours, il faut même pouvoir se transformer. Les discours de "réinvention" sont potentiellement plus émergents et négociés qu'ils ne pourraient le sembler a priori. En habilitant des énoncés de confession, bien que dégradants et avilissants, les discours de la réinvention ne permettent-ils pas à certains acteurs et à des organismes du secteur public d'intéresser les gouvernements et de justifier, c'est le cas dans le secteur de la culture, des investissements colossaux?

Selon nous, la réinvention des musées de la nature, des musées de science et des centres de science est à l'appui d'une conception du musée comme producteur actif de patrimoine. En ce sens, le musée est un laboratoire des expériences civiques ${ }^{[37}$. Il donne la possibilité de gérer et de resocialiser les objets techniques, la nature et la science en son enceinte.

\section{[36]}

FOUCAULT, Michel.

Power/Knowledge: Selected Interviews and Other Writings, 1972-1977. New York : Pantheon Books, 1980. [37]

LATOUR, Bruno et Steve Woolgar. Laboratory Life. The Construction of Scientific Facts. Princeton NJ : Princeton University Press, 1986. Dans une perspective plus muséologique, on peut également lire Bennett, Tony. "Civic Laboratories: Museums, Cultural Objecthood, and the Governance of the Socialn. Centre for Research on Sociocultural Change. Londres : The Open University, 2005. 
[38]

CALLON, Michel.

"Éléments pour une sociologie de la traduction : La domestication des coquilles Saint-Jacques et des marins-pêcheurs dans la baie de Saint-Brieuc". L'année sociologique, vol. 36,

SCHIELE, Bernard. Le musée de sciences. Montée du modèle communicationnel et recomposition du champ muséal. Paris : L'Harmattan, 2001.
Développée par Michel Callon ${ }^{\text {[38 }}$ et Bruno Latour, la sociologie de la traduction semble être un modèle qui permet de rendre compte des changements actuels au sein des musées de science. Liant pouvoir et identité, la sociologie de la traduction invite à analyser les discours et les pratiques afin de dégager les stratégies d'acteur qui permettent à ces derniers de justifier leur pertinence et de négocier leur identité afin de devenir les indispensables points de passage obligés. Stratégie d'action collective, la réinvention des musées semble attester d'un désir de devenir tantôt représentant de l'innovation, tantôt représentant du dialogue science / société. Témoignant des pressions multidirectionnelles auxquelles les musées doivent faire face dans le présent, les discours de réinvention annoncent des mutations institutionnelles qui ne sont pas conséquences pour les pratiques de la muséologie des sciences. Au-delà de ces grands discours de renouvellement institutionnel, il faut saisir également la transformation identitaire telle qu'elle se dessine actuellement dans l'émergence des pratiques qui métissent communication, démocratie et apprentissage. L'avènement des réinventions institutionnelles laisse alors place à la prolifération des pratiques expertes aux pratiques d'évaluation d'impact qui laissent croire à plusieurs transformations à venir de la fonction recherche des musées, délaissant l'objet au profit de l'étude du social axée sur les pratiques des sciences de l'éducation et de la communication délibérative. Tel qu'annoncé par Bernard Schiele, nous assistons ici à une radicalisation et à un approfondissement des logiques du modèle communicationnel ${ }^{\left[{ }^{[3]}\right.}$. À l'instar des musées européens, les musées de science canadiens semblent poursuivre et multiplier les initiatives de nature communicationnelle.

Une fois de plus, dirons-nous que le patrimoine scientifique n'est pas que le témoignage du talent et de l'excellence qu'il suffirait de cueillir. Fruit de stratégies, de traductions, de relectures identitaires, il est construit dans les luttes de pouvoir, sur le tissu sociopolitique des rapports État / culture. Comme tous les patrimoines, le patrimoine scientifique est le produit de détours, de contestations et d'alliances, bref d'un champ de bataille si caractéristique de ce que Latour qualifierait probablement d'une culture en train de se faire. 
Scientific Policies and the Transformation of Institutional Projects in Technical and Scientific Museums: A discourse of reinvention

\section{in perspective}

[translated by Allana Carlyle]

"The province is delighted with the Science Centre's change in philosophy..." ${ }^{[40]}$

Owing to the transition from scientific policy to a formal innovation policy in 2002, the field of "scientific culture" in Canada has lost what was left of its legitimacy. In fact, a large funding program, Science and Culture Canada, was cancelled in 1999 , dealing a hard blow to the field's professionals. But that shift is not unique to Canada. At the end of the 1990s, "scientific culture" was no longer on the agenda. We are in fact experiencing a shift from a "scientific culture" to an "innovation culture." While many OECD countries are launching into the creation of innovation policy and leaving aside traditional scientific policies, the relationship between science and society is taking a different turn. Through the 1990 s to the year 2000 , we have also experienced an increase of mediatisation regarding concerns for technosciences. From the highly optimistic Vancouver Declaration in 1989 to the new Science Agenda for UNESCO in 1999, it will have taken but ten years to alter the public's attitude towards sciences. In that context we can observe the emergence of a confirmed governmental interest in science museums. Motivated by a more ambiguous relationship between science and society, many countries are indeed moving towards the creation of science museums, nature museums, and science centres in order to give rise to diverse avenues of communication.

Stemming from a wave of administrative transformations throughout the public sector, cultural institutions are in a state of unrest. More specifically, since the 1980s, museums have been subjected to a multitude of managerial discussions focusing on the practices and institutional projects of public sector museums. For a number of years, many such discussions have called for the changing, the renewal, and the transformation of large cultural institutions. Those administrative reforms are accompanied by a flood of discourse calling for, and even celebrating the change in the public sector. ${ }^{[41]}$

\section{[40]}

DI COCCO, Caroline

Ontario Minister of Culture.

Media release, July 26, 2006

[41]

POLLITT, Christopher et Geert Bouckaert. Public Management Reform. A Comparative Analysis. Oxford: Oxford University Press, 2004, p. 18. [42]

FAIRCLOUGH, Norman. Critical Discourse Analysis. The Critical Study of Language. Londres : Longman, 1995. 
In certain science museums and public science centres in Ontario and some at a federal level in Canada, the emergence of an atmosphere of change is experienced. As found in the famous call for change by Osborne and Gaebler in 1992 (Reinventing Government), in the large museums of science and technology discussions suggest the major transformation of these institutions: Renaissance ROM (Royal Ontario Museum), The Big Change (Science North) Renewal (Canadian Museum of Nature) The Agents of Change Initiative (Ontario Science Centre). Through the

SCHIELE, Bernard. Le musée de sciences. Montée du modèle communicationnel et recomposition du champ muséal. Paris : L'Harmattan, 2001. analysis of institutional discourse, ${ }^{\left[{ }^{[2]}\right]}$ based on a literature review, this article will present an interpretation of the new relationships being woven between the State, the museum, and scientific heritage.

We analyzed the multidirectional discourse in order to measure changes in sciences and in managerial attitudes. Two visions particularly attracted our attention: the managerial "confession" and the scientific "conversion." As described by Schiele in $2001,{ }^{[43]}$ we are now participating in the strengthening and the deepening of the communication model's rationale. While science centres are fostering a culture of innovation, nature museums seem to choose the path of dialogue between science and nature. Following the example of European science museums, but with certain nuances, the Canadian science museums examined seem to come close to the communication ideals of deliberative democracy. 